\title{
Representación espacial de zonas de recarga del agua subterránea a partir de mapas isotópicos de precipitación. Caso de estudio: Valle de Aburrá, Colombia
}

\author{
Juliana Ossa, Ana Karina Campillo, Cristian Omar y Teresita Betancur \\ Universidad de Antioquia. Calle 67 \#53-108, Medellín, Colombia. \\ juliana.ossav@udea.edu.co; anakarinacp21@gmail.com; \\ cristian.omar@udea.edu.co; teresita.betancur@udea.edu.com
}

\begin{abstract}
RESUMEN
Los isótopos ambientales constituyen una herramienta para interpretar los procesos hidrológicos relacionados con los ambientes subterráneos. Estos se han utilizado para la validación de modelos hidrogeológicos conceptuales, en términos de las zonas de recarga, tránsito y descarga, así como la identificación de interacciones con otros cuerpos de agua y determinación del tiempo de residencia del agua en las unidades hidrogeológicas. La determinación de los procesos de recarga ha sido usualmente validada mediante el conocimiento de los isótopos estables ${ }^{18} \mathrm{O}$ y ${ }^{2} \mathrm{H}$. A nivel regional esta evaluación se ha abordado mediante la construcción de mapas isotópicos, combinado con un entendimiento de las características hidrológicas presentes en la zona de estudio y el conocimiento de modelos hidrogeológicos conceptuales. Este trabajo permitió desarrollar un esquema para el análisis de las potenciales áreas de recarga de agua subterránea a partir de la variabilidad espacial de ${ }^{18} \mathrm{O} y^{2} \mathrm{H}$ en la precipitación, el flujo de base y el agua subterránea mediante la construcción de mapas isotópicos, utilizando herramientas de modelación espacial. A partir de un trabajo preliminar realizado para el Departamento de Antioquia, se retomó un área estratégica en la que los resultados son más contundentes y tienen mayores implicaciones en materia de gestión del agua subterránea: el Valle de Aburrá-Colombia
\end{abstract}

Palabras clave: Acuíferos, agua subterránea, isotopía, modelación espacial, recarga.

\section{Recharge área maps from precipitation isoscapes. Case study: Aburrá Valley, Colombia}

\begin{abstract}
The environmental isotopes are a tool for interpreting the hydrological processes related to underground environments. These are used for the validation of hydrogeological models, in terms of recharge, transit and discharge zones, as well as the identification of groundwater interactions with other water bodies and groundwater residence time in the hydrogeological units. The determination of the recharge processes have usually been validated from knowledge about the stable isotopes present in the water molecule $\left({ }^{18} \mathrm{O}\right.$ and $\left.{ }^{2} \mathrm{H}\right)$. At the regional level, this evaluation has been addressed through the construction of isoscapes combined with an understanding of the hydrological characteristics present in the study area and knowledge of conceptual hydrogeological models. The objective of this study is to apply a scheme for the analysis of potential groundwater recharge zones, from ${ }^{18} \mathrm{O}$ and ${ }^{2} \mathrm{H}$ spatial variability in precipitation, baseflow and groundwater, using spatial modelling tools. Based on a preliminary work done for the Antioquia department, a strategic area was chosen in which the results are more forceful and have greater implications in groundwater management: the Aburrá Valley-Colombia.
\end{abstract}

Keywords: aquifer, groundwater, isotopy, spatial modeling, recharge. 


\section{Introducción}

Los isótopos ambientales son una herramienta para la interpretación de los procesos hidrológicos relacionados con los ambientes superficiales y subterráneos. El uso de los isótopos que componen la molécula del agua $\left({ }^{18} \mathrm{O}\right.$ y $\left.{ }^{2} \mathrm{H}\right)$ permite pensar en diferentes rutas del agua en el ciclo hidrológico (Craig 1961, Dansgaard 1964) y su uso en la hidrogeología parte del hecho que se pueden tener diferentes composiciones isotópicas de acuerdo con el ambiente de recarga. La relación de isótopos entre aguas superficiales y subterráneas, la similitud en la composición entre las aguas de precipitación y las tomadas del acuífero, la relación altitudinal y la semejanza entre aguas provenientes de formaciones vecinas, permiten interpretar el fenómeno de la recarga, determinar patrones de flujo regional y confirmar la distribución de las unidades hidrogeológicas (Clark \& Fritz, 1997). Teniendo en cuenta las diferentes rutas y los procesos a los que están sometidos estos isótopos durante el ciclo hidrológico y estableciendo relaciones con las diferentes condiciones climáticas de cada zona de estudio, es posible entender la dinámica de la precipitación y su relación con las aguas subterráneas, para determinar principalmente los mecanismos y zonas de recarga mediante la construcción de isoscapes (Sánchez-Murillo y Birkel, 2016).

El término (neologismo) anglosajón "isoscapes" es la contracción de isotope-landscape. La traducción española puede ser mapas isotópicos. Se refiere al mapeo de las distribuciones espaciotemporales a gran escala de las proporciones de isótopos estables en diversas matrices o reservorios ambientales, como la lluvia, los océanos, las rocas, las plantas y los animales (Wassenaar et al., 2009). Estas técnicas han sido beneficiadas por los software de sistemas de información geográfica y la medición a lo largo del tiempo de datos isotópicos (Wassenaar et al., 2009). En el caso de los isótopos estables de la molécula del agua, los mapas isótopicos constituyen una herramienta para determinar a escala regional las zonas de recarga y posteriormente establecer mecanismos de gestión frente a estas. Sin embargo, para poder utilizar estos mapas isotópicos como herramienta de gestión es necesario mejorar la resolución espacial y adaptarla a las escalas regionales y locales, que invocan más esfuerzos de muestreo para captar las variaciones espaciotemporales, particularmente en los paisajes montañosos, debido a su inherente complejidad ambiental (Yamanaka et al., 2015 y Langston et al., 2011).

En Colombia, las aguas subterráneas tienen importancia significativa en cerca del $75 \%$ del territorio. Sin embargo, menos del $15 \%$ están siendo exploradas en términos de establecer balances de agua adecuados y modelos hidrogeológicos conceptuales. De 61 sistemas hidrogeológicos de interés que han sido identificados, sólo 16 cuentan con estudios de detaIle (IDEAM, 2018). Por lo tanto, es necesario aunar esfuerzos en los niveles nacionales y regionales, en la exploración y en la caracterización de los sistemas de agua subterránea, para asegurar su protección, particularmente en las zonas de recarga.

Ossa et al. (2018) realizaron una síntesis de la información isotópica de agua lluvia y agua subterránea en un área de $63000 \mathrm{~km}^{2}$ al noroccidente de Colombia - departamento de Antioquia. Para esta región obtuvieron una Línea Meteórica Regional -LMRA-, cuya ecuación es $\delta^{2} \mathrm{H}=8,09 \delta^{18} \mathrm{O}+10,9 \%$. A partir del análisis de datos provenientes de información de precipitación de distintas zonas, incluyendo áreas costeras, piedemontes, cordillera y valles, se estableció un gradiente altitudinal de $-0,17 \%$ o $\delta^{18} \mathrm{O}$ cada $100 \mathrm{~m}$, con el cual se construyó un mapa de distribución espacial de la composición isotópica en la precipitación. Adicionalmente se avanzó en una versión preliminar de un modelo regional para la relación precipitación-recarga para el departamento. Este ejercicio se refinó a nivel local para la región del Valle de Aburrá.

El propósito del presente trabajo fue el de evaluar a partir de mapas isotópicos de precipitación y aguas subterráneas las potenciales zonas de recarga del sistema acuífero del Valle de Aburrá (Antioquia-Colombia), con el fin de determinar el origen del agua que sostiene los niveles subterráneos, en una zona donde las superficies de recarga directa han alcanzado un cambio en la cobertura por efectos de la urbanización, reduciéndose en más de $74 \%$ la superficie de recarga directa inicial.

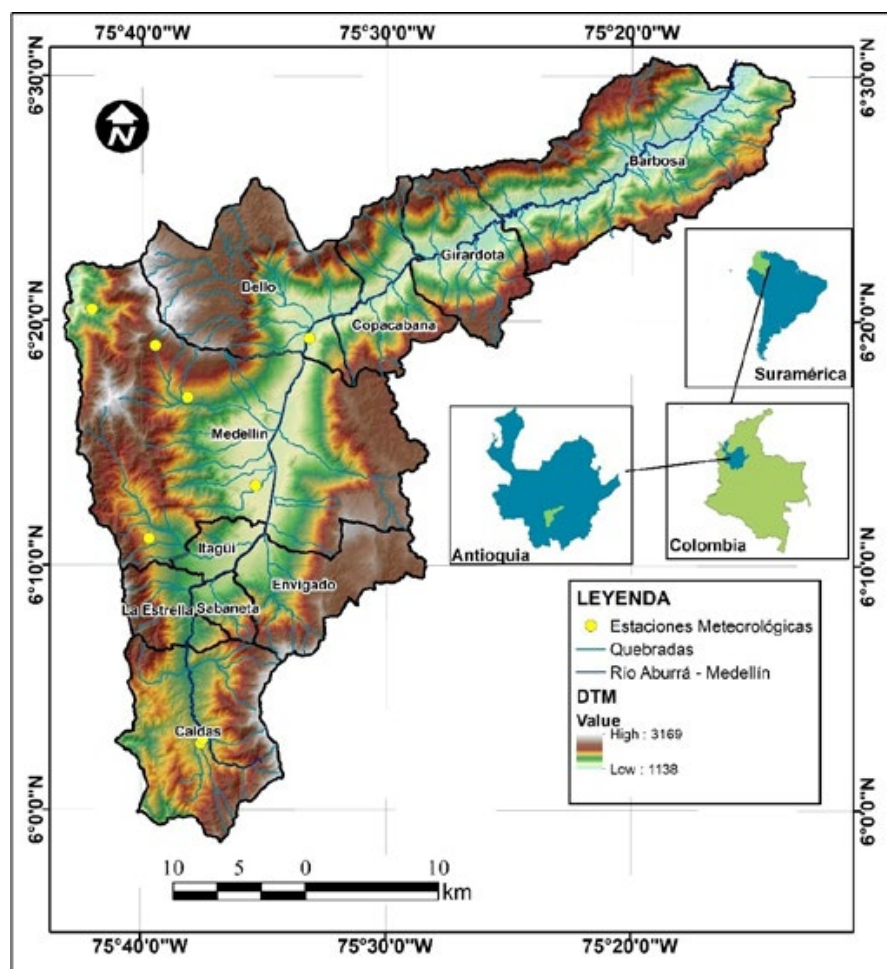

Figura 1. Localización de la zona de estudio.

Figure 1. Location of the study zone. 


\section{Descripción del área de estudio}

El Valle de Aburrá (Figura 1) tiene una extensión de $1152 \mathrm{~km}^{2}$. Las montañas que lo limitan tienen una altura promedio de $2700 \mathrm{~m}$ s.n.m, el punto más bajo se encuentra a $1138 \mathrm{~m}$ s.n.m y el de mayor elevación a 3169 m s.n.m. La temperatura oscila entre los 16 y $29^{\circ} \mathrm{C}$. La precipitación promedio anual es de 1500 $\mathrm{mm} / a n ̃ o$, registrándose valores máximos de 3500 $\mathrm{mm} / a n ̃ o$. El régimen de lluvia es bimodal, con dos épocas lluviosas (de abril a junio y septiembre a noviembre) y dos épocas secas (diciembre a marzo y junio a agosto). En esta región se han identificado 3 unidades hidrogeológicas de carácter acuífero: i) el Acuífero Libre de Valle de Aburrá, que se extiende a lo largo de todo el valle, ii) el Acuífero Semiconfinado del Valle de Aburrá y iii) el Acuífero de la Dunita de Medellín, que constituye una unidad acuífera dado el grado de fracturación de esta unidad de roca, sumado a la condición de pseudokarst (Área Metropolitana del Valle de Aburrá y Universidad de Antioquia, 2013).

Si bien los 10 municipios que conforman el Valle de Aburrá tienen una amplia cobertura en el suministro de agua potable, las fuentes superficiales de las que se abastece la población, cercana a los 3,5 millones de habitantes, es importada de otras cuencas. El agua subterránea en este territorio tiene un valor estratégico como fuente complementaria o alternativa, ante posibles escenarios de escasez por variabilidad o cambio climático y en virtud de la función ecosistémica de soporte a cuerpos de agua superficiales, como ríos, corrientes y humedales.

\section{Metodología}

\section{Hidrogeología del Valle de Aburrá}

En el modelo hidrogeológico conceptual del Valle de Aburrá (Figura 2), se tienen delimitadas las unidades hidrogeológicas consideradas acuíferos y las rocas encajantes que actúan como fuente de recarga indirecta al configurar sistemas de flujo intermedios $y$ regionales. El acuífero libre del Valle de Aburrá, con una extensión superficial de $238,6 \mathrm{~km}^{2}$, está conformado por depósitos aluviales del río Medellín y sus principales afluentes y por varios depósitos de vertiente que poseen matriz limo-arenosa. El espesor de esta unidad alcanza los $150 \mathrm{~m}$ y la conductividad hidráulica alcanza $50 \mathrm{~m} /$ día en el centro del Valle. En el centro y sur de la zona de estudio, separado por una capa discontinua de carácter arcilloso, por debajo del Libre, se encuentra el Acuífero Semiconfinado, el cual alcanzaría profundidades superiores a $207 \mathrm{~m}$. La Dunita de Medellín, roca ultramáfica serpentinizada, ha alcanzado importantes niveles de disolución, a través de grietas y fracturas; por esta razón se le ha atribuido la condición de pseudokarst; los caudales en los manantiales que en ella se presentan (del orden de $12 \mathrm{~L} / \mathrm{s}$ ) llevan a que este cuerpo litológico sea consi- derado como un acuífero y que se haya propuesto su exploración futura. El dominio encajante de los acuíferos del Valle de Aburrá está conformado por un sistema montañoso emplazado sobre rocas ígneas y metamórficas, de edades Paleozoico y posteriores. Estas rocas están afectadas estructuralmente por sistemas de fallas regionales que cruzan el territorio en sentido NS a NNW al oeste del río Aburrá y una tendencia NW al este del mismo; ello les imprime una fuerte afectación estructural, que se manifiesta en sistemas de fracturas y diaclasas que, en muchos casos, imprimen a la litología una porosidad secundaria.

De acuerdo con Escobar et al. (2017) y Betancur et al. (2017), el sistema acuífero del fondo del Valle estaría recibiendo aportes de flujos intermedios y regionales desde las rocas encajantes del sistema. Además de las zonas de recarga directa, se han delimitado - a partir de la combinación de criterios geomorfológicos, estructurales, tipo de suelo y capacidad de infiltración - zonas de recarga indirecta con distintos grados jerárquicos de importancia: alta, media o baja.

Desde el año 2010, el Área Metropolitana del Valle de Aburrá -AMVA- viene implementando y fortaleciendo una red de monitoreo hidrogeológico, que opera con la Universidad de Antioquia. La red, que en principio solo fue piezométrica, prontamente empezó a incluir análisis hidrogeoquímicos y cuenta, desde 2017, con puntos de monitoreo isotópico, tanto para agua Ilu-

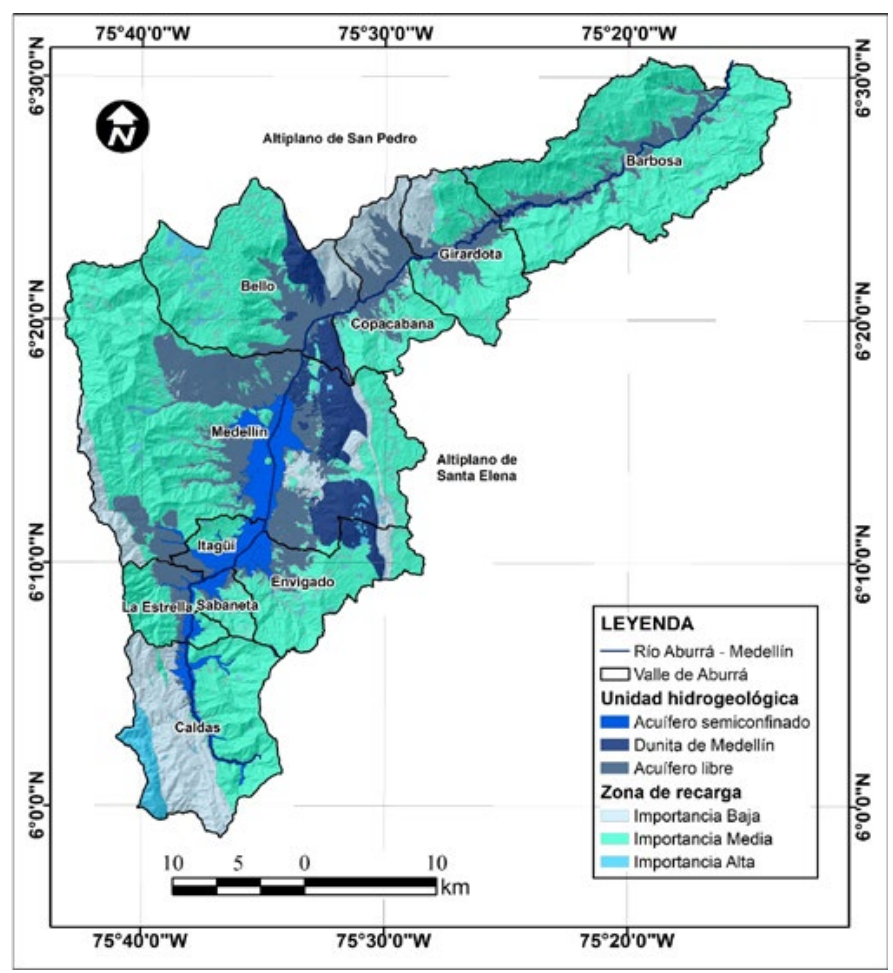

Figura 2. Unidades hidrogeológicas y zonas de recarga del sistema acuífero del Valle de Aburrá. (Fuente: Escobar et al., 2017).

Figure 2. Hydrogeological units and recharge areas of the Aburrá Valley aquifer. (Source, Escobar et al, 2017). 


\begin{tabular}{|c|c|c|c|c|c|}
\hline Z.E & Análisis & Tipo & Cant. & Periodo & Fuente \\
\hline \multirow{6}{*}{$\begin{array}{l}\text { Valle } \\
\text { Aburrá }\end{array}$} & \multirow{3}{*}{$18 \mathrm{O}$ у $2 \mathrm{H}$} & Agua Iluvia & 180 & 2011- 2018 & \multirow{3}{*}{$\begin{array}{l}\text { Área Metropolitana del Valle de Aburrá \& } \\
\text { Universidad de Antioquia, 2013; Campillo, } \\
\text { 2012; Cano, 2015, IAEA, } 2017\end{array}$} \\
\hline & & Agua subterránea & 124 & 2011- 2018 & \\
\hline & & Agua superficial & 29 & 2011- 2018 & \\
\hline & \multirow[b]{2}{*}{$3 \mathrm{H}$} & Agua Iluvia & 1 & \multirow[b]{2}{*}{2015} & \multirow{2}{*}{$\begin{array}{l}\text { Área Metropolitana del Valle de Aburrá \& } \\
\text { Universidad de Antioquia, } 2017\end{array}$} \\
\hline & & Agua subterránea & 6 & & \\
\hline & $14 C$ y $13 C$ & Agua subterránea & 4 & 2018 & $\begin{array}{l}\text { Area Metropolitana del Valle de Aburrá \& } \\
\text { Universidad de Antioguia, } 2018\end{array}$ \\
\hline
\end{tabular}

Tabla 1. Síntesis de información isotópica disponible para el Valle de Aburrá.

Table 1. Synthesis of available isotopic information of Aburrá Valley.

via como superficial y subterránea. En la figura 3 se muestra la superficie piezométrica promedio del acuífero libre obtenida a partir de la red de monitoreo del AMVA. De acuerdo con ella, el flujo subterráneo se orienta desde los límites laterales del acuífero, en las áreas de contacto con la roca encajante, hacia el centro del valle.

Esfuerzos puntuales habían sido realizados antes para tratar de establecer una relación entre la precipitación en las vertientes y la parte alta de la cordillera con el agua disponible en el acuífero (Campillo, 2012, Cano, 2015, Área Metropolitana del Valle de Aburrá y Universidad de Antioquia, 2013). En 2017, mediante la realización del proyecto "Analysis of Groundwater Recharge from Isoscapes of Precipitation and Shallow

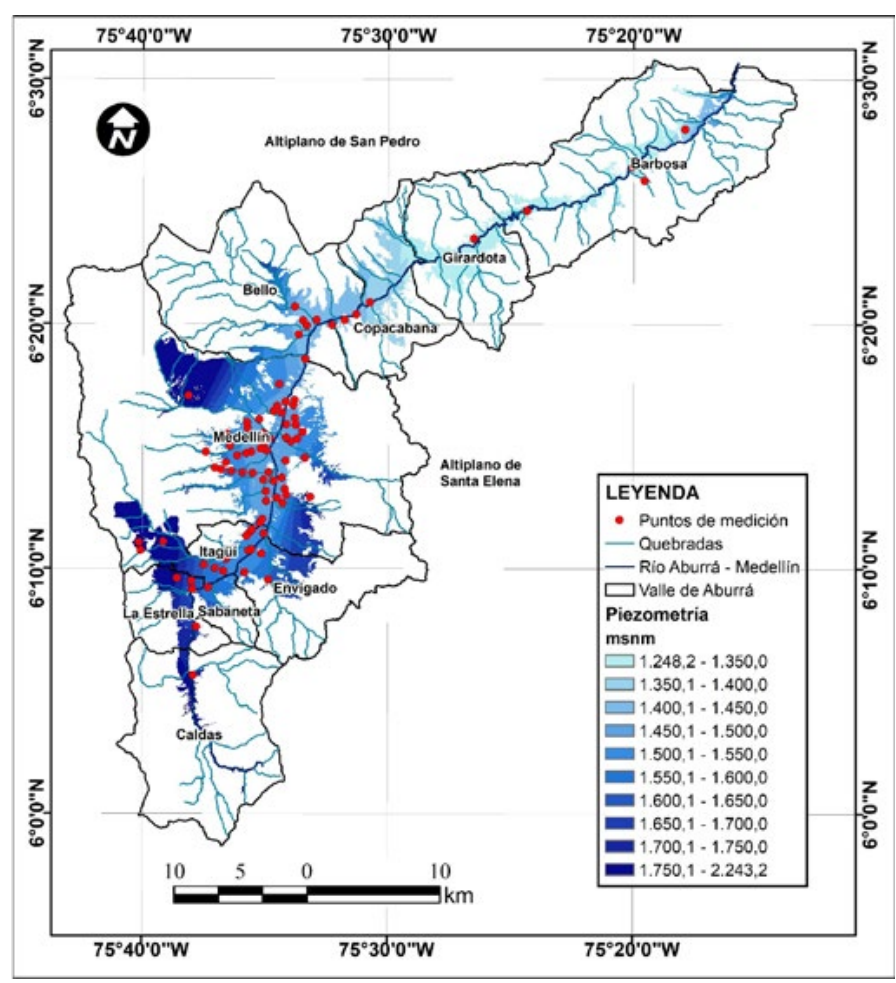

Figura 3. Superficie piezométrica del Acuífero Libre del Valle de Aburrá.

Figure 3. Piezometric surface of the phreatic Aquifer of the Valle de Aburrá.
Groundwater in the Northwest of Colombia", con el apoyo del Organismo Internacional de Energía Atómica -OIEA se construyó el primer mapa isotópico para Antioquia y se evaluaron las primeras relaciones con la recarga de algunos sistemas acuíferos de la región (Betancur et al, 2017 y Ossa et al., 2018).

\section{Datos y fuentes de información disponible}

En la Tabla 1 se resume la información isotópica disponible para el Valle de Aburrá, indicando las fuentes de la misma y los laboratorios en los cuales se efectuaron los análisis.

Se tuvieron ocho (8) estaciones de agua lluvia, distribuidas desde el fondo ( $1450 \mathrm{~m}$ s.n.m) hasta una altura de $2500 \mathrm{~m}$ s.n.m, procurando una localización altitudinal a intervalos de $250 \mathrm{~m}$, y si era posible en ambas vertientes del valle. De esta forma se logró establecer una adecuada representatividad espacial. Durante el periodo de muestreo, en cada estación se dispusieron totalizadores desde los cuales se recuperaba mensualmente el agua precipitada y se tomaban $60 \mathrm{~mL}$ que eran almacenados en frascos de vidrio ámbar con tapa y contratapa evitando la presencia de burbujas o cámaras de aire; durante la toma de cada muestra se contabilizaba el volumen de agua precipitada durante el mes. Los períodos durante los que se efectuó el muestreo corresponden a años hidrológicos normales. Los muestreos de agua subterránea provienen de 45 pozos, en los que se tomaron muestras en tres momentos diferentes. De acuerdo con los análisis hidrogeoquímicos practicados durante la operación de la red de monitoreo, se seleccionaron 4 puntos que captan el acuífero libre para efectuar análisis de $3 \mathrm{H}$. La distribución de los sitios de monitoreo isotópico se presenta en la Figura 4.

\section{Tratamiento de la información}

Dentro de los tratamientos dados a la información se obtuvo, a partir de la precipitación, la Línea Meteórica Local ( $L M L)$, se verificaron los efectos que determinan el fraccionamiento isotópico (altitudinal, cantidad y estacionalidad). También se estableció la relación de la composición isotópica con el agua subterránea (Mook, 2002). 


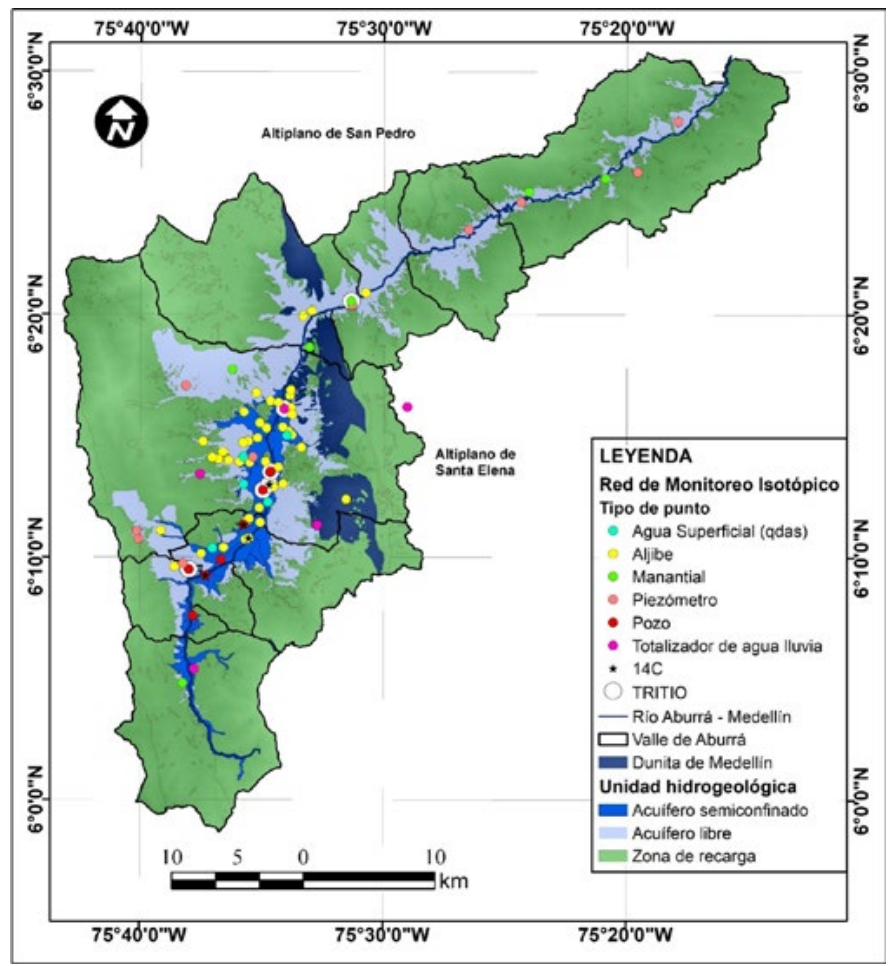

Figura 4. Red de monitoreo isotópico en el Valle de Aburrá. Figure 4. Isotopic monitoring network in the Aburrá Valley.

Teniendo en cuenta la composición isotópica del agua lluvia, en consideración a la cota topográfica en la que se localizan las estaciones de toma de muestras, se calculó el gradiente altitudinal y se estableció la relación lineal que lo define. Esta determinación serviría como un primer punto de referencia para empezar a establecer, a partir de la información de composición isotópica del agua subterránea, la comparación que indica las probables alturas en las que se dieron las lluvias que luego recargarían al acuífero (Gonfiantini et al., 2001; Baudry et al., 2018).
A partir de la ecuación del gradiente altitudinal obtenido y usando los datos de $\delta^{18} \mathrm{O}$ y $\delta^{2} \mathrm{H}$, tomando como base el modelo digital de elevación de $12,5 \mathrm{~m}$ por $12,5 \mathrm{~m}$, disponible a partir de los datos del radar Alos PALSAR de la agencia espacial JAXA (C) JAXA/ METI [2018], se calculó el mapa de isotópico de precipitación para el Valle de Aburrá.

Luego de explorar varios modelos de análisis espacial se aplicó el método de Ponderación de la Inversa de la Distancia (Londoño et al., 2010) para representar la variación espacial de los datos de $\delta^{18} \mathrm{O}$ y $\delta^{2} \mathrm{H}$ obtenidos para el agua subterránea.

De acuerdo con Sánchez-Murillo y Birkel, (2016), con el fin de evaluar los mecanismos de recarga, a partir de la calculadora raster de ArcGis se evaluó la relación (P/AS) entre los mapas de la composición isotópica de la precipitación ( $P$ ) y el agua subterránea (AS). Si de esta relación se obtiene un valor menor que 1, se infiere la posibilidad de que las zonas de recarga se encuentran a una altura mayor de donde se toma la medición; si la relación es igual a 1 significa que el agua que se recarga provendría de lluvias locales, produciéndose una recarga directa; y si la relación es mayor que 1 significa que se podrían estar presentando procesos de evaporación o que el agua se habría recargado en condiciones climáticas diferentes. Estos mapas fueron generados con información agregada a escala anual.

Posteriormente se realizaron ejercicios de superposición espacial, para analizar la relación entre los resultados de áreas de recarga sugeridos por la isotopía y aquellas delimitadas en el modelo hidrogeológico conceptual (Escobar et al., 2017 y Betancur et al., 2017).

Para tratar de soportar con más evidencias los resultados alcanzados, se utilizaron los resultados de 5 análisis de tritio, uno en agua lluvia y cuatro en aguas provenientes del acuífero libre. Si los valores reportados para tritio en el acuífero eran menores a 0,8 UT, se consideraba -teniendo en cuenta el límite de detección del laboratorio y la composición de la lluvia- que las aguas subterráneas tendrán una edad de más de 60 años.

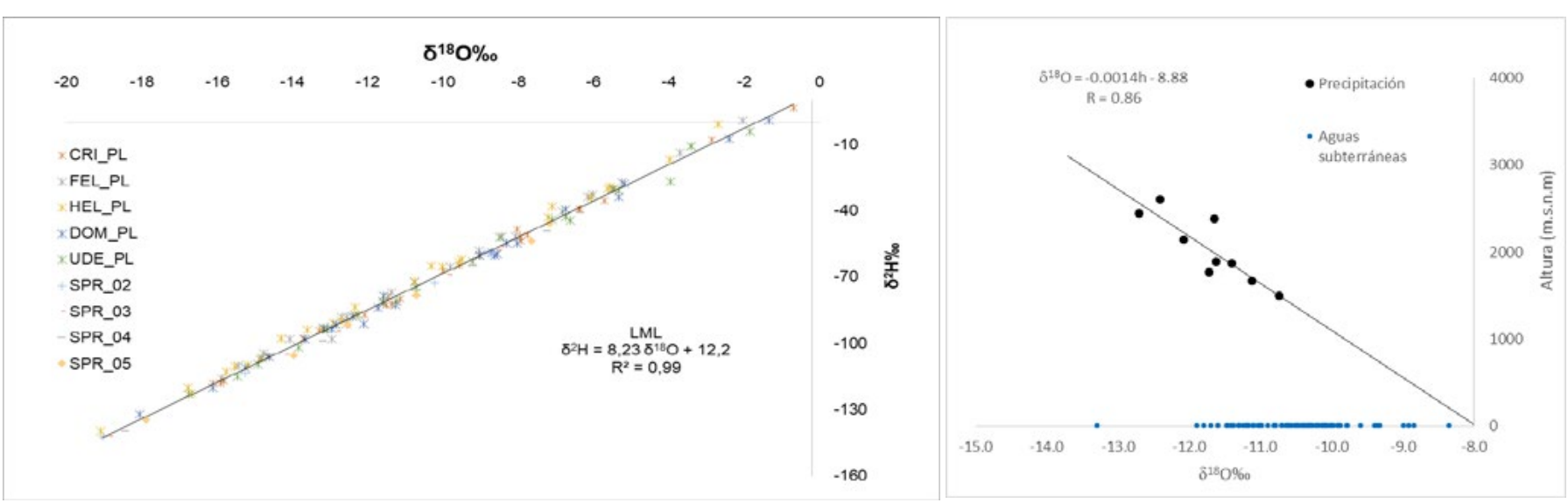

Figura 5. Línea Meteórica Local-LML del Valle de Aburrá y gradiente altitudinal para el d ${ }^{18} \mathrm{O} \%$.

Figure 5. Local meteoric line (LML) of Valle de Aburrá and altitudinal relation. of $\delta^{18} \mathrm{O} \%$. 


\section{Resultados}

En la tabla 2 se presentan los datos de $\delta^{18} \mathrm{O}$ y $\delta^{2} \mathrm{H}$, utilizados para evaluar las características isotópicas del agua lluvia en el Valle de Aburrá. A partir de ellos se determinó la línea meteórica local que corresponde a la siguiente ecuación $\delta^{2} \mathrm{H}=8,23 \delta^{18} \mathrm{O}+12,2 \%$ (figura 5), similar a la Línea Meteórica Mundial (Mook, 2002). Además, fueron verificados los efectos de estacionalidad y de cantidad, que son muy importantes en las regiones tropicales. En el Valle de Aburrá es posible verificar el régimen bimodal, presentándose valores más enriquecidos en la época seca (diciembre a marzo y junio a agosto) y valores más empobrecidos durante las temporadas húmedas (abril a junio y septiembre a noviembre). El gradiente altitudinal que se obtiene para el valle es de $-0,14 \%$ on $\delta^{18} \mathrm{O}$ por cada 100 metros (figura 5). La tendencia del descenso se ajusta a la ecuación $\delta^{18} \mathrm{O}=-0,0014 \mathrm{~h}-8,837$, la cual refleja claramente la variación lineal normal del gradiente con un ajuste representado en un valor de $R=0,86$. Esta magnitud del gradiente es coherente con los que se obtienen en otras regiones montañosas de Colombia (Gómez et al., 2015) y en regiones montañosas tropicales (Gonfiantini et al., 2001). Aplicando sobre el modelo digital de elevación la relación que se obtiene a partir de este gradiente, se obtiene el mapa isotópico de $\delta^{18} \mathrm{O}$ en la precipitación para el Valle de Aburrá (Figura 6). Los valores calculados del $\delta^{18} \mathrm{O}$ están en el intervalo desde $-12,18 \%$ o de $\delta^{18} \mathrm{O}$, en las zonas más altas, hasta $-9,94 \%$, en las más bajas, lo que corresponde con un rango de variación del $\delta^{18} \mathrm{O}$ de $2,24 \%$.

Como resultado de la interpolación espacial de los registros para $\delta^{18} \mathrm{O}$, obtenidos a partir de las muestras de agua tomadas del acuífero libre se obtiene el modelo digital que se presenta en la Figura 6. El intervalo de variación resultante tiene un rango de $3,24 \%$, para un intervalo de valores que oscilan entre $-11,90 \%$ o hasta $-8,66 \%$. La variabilidad espacial resultante está representada en la identificación de zonas en las que se observa una relativa homogeneidad en la naturaleza del agua subterránea, pudiendo -si se quiere- delimitar polígonos en los que se registran variaciones no mayores de $0,36 \%$, condición que debe tener un significado especial para explicar las fuentes o zonas de recarga.

Mediante la relación P/AS se obtiene el mapa de la Figura 8. Como se mencionó anteriormente, esta relación permite identificar mecanismos de recarga. A partir del análisis estadístico de los datos isotópicos de precipitación, en los que se consideró el promedio, la media, la mediana y la desviación estándar, en este trabajo se estableció como valor de 1 para la relación P/AS el intervalo $(0,98$ a 1,02$)$.

Para el Acuífero Libre en el fondo Valle de Aburrá la extensión de la superficie en la que relación P/AS es menor a 1, permite identificar que efectivamente se registra recarga relacionada con precipitaciones

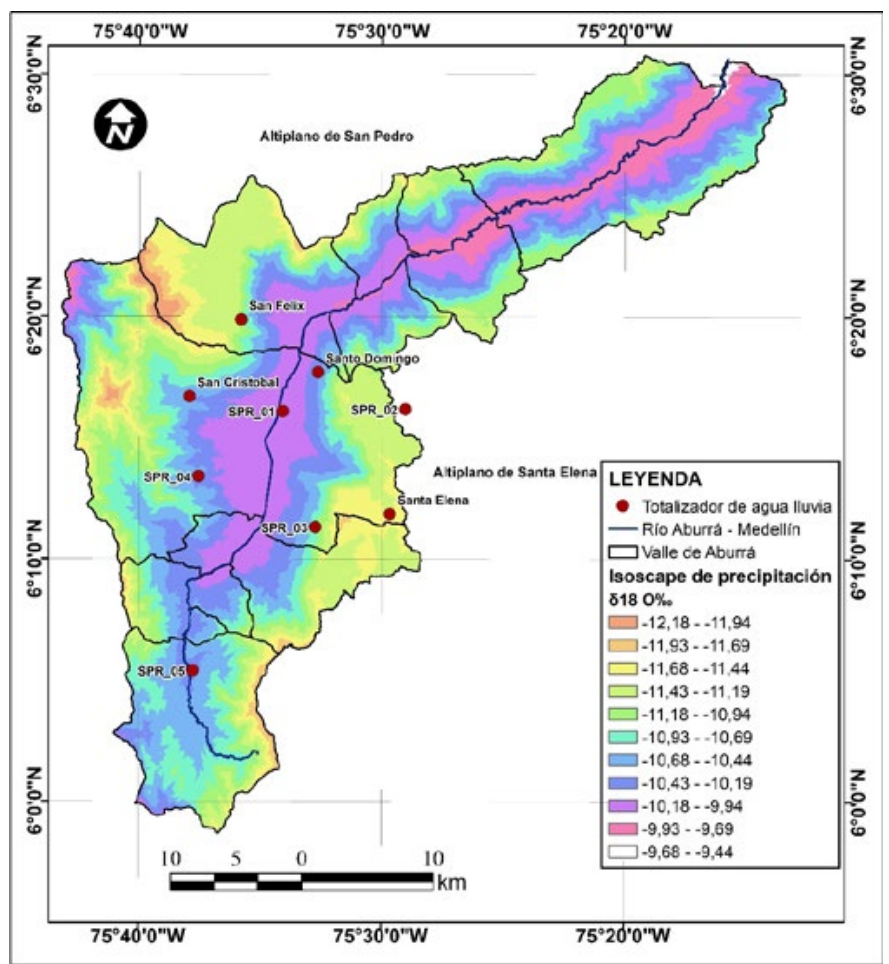

Figura 6. Mapa isotópico de $\delta^{18} \mathrm{O} \%$ en la precipitación para el Valle de Aburrá.

Figure 6. Isoscape of $\delta^{18} \mathrm{O} \%$ of the Aburrá Valley precipitation.

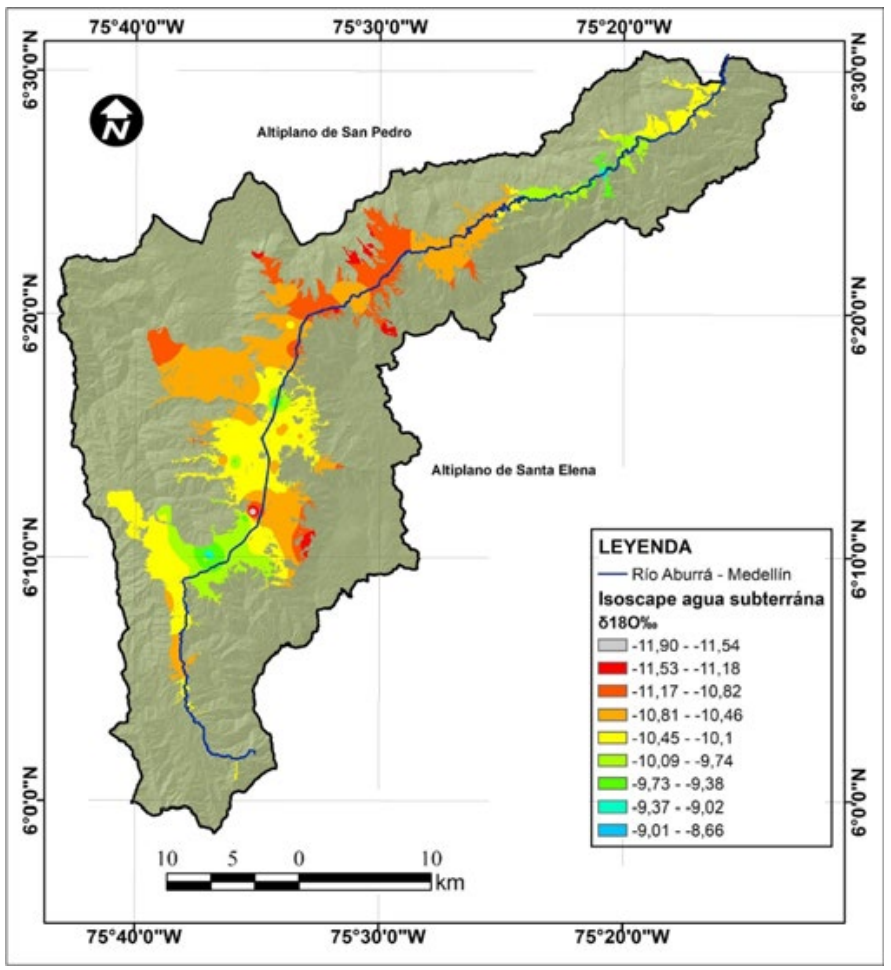

Figura 7. Distribución espacial del $\delta^{18} \mathrm{O} \%$ para el acuífero libre del Valle de Aburrá.

Figure 7. Spatial distribution of ${ }^{18} \mathrm{O} \%$ of the Aburrá Valley aquifer. 


\begin{tabular}{|c|c|c|c|c|c|c|c|c|c|}
\hline Código & Nombre & $\mathbf{X}$ & $\mathbf{Y}$ & Cota & tipo de muestra & Fecha & $18^{\circ}$ & $2 \mathrm{H}$ & d \\
\hline CRI_PL & San Cristóbal & 827938.3 & 1186292.7 & 1870.5 & Lluvia & mar-11 & -8.84 & -60.2 & 10.5 \\
\hline CRI PL & San Cristóbal & 827938.3 & 1186292.7 & 1870.5 & Lluvia & $a b r-11$ & -14.66 & -107.2 & 10.1 \\
\hline CRI_PL & San Cristóbal & 827938.3 & 1186292.7 & 1870.5 & Lluvia & may-11 & -15.91 & -118.1 & 9.2 \\
\hline CRI_PL & San Cristóbal & 827938.3 & 1186292.7 & 1870.5 & Lluvia & jun-11 & -15.61 & -117.2 & 7.7 \\
\hline CRI_PL & San Cristóbal & 827938.3 & 1186292.7 & 1870.5 & Lluvia & jul-11 & -11.31 & -82.6 & 7.9 \\
\hline CRI_PL & San Cristóbal & 827938.3 & 1186292.7 & 1870.5 & Lluvia & ago-11 & -11.88 & -87.2 & 7.8 \\
\hline CRIPL & San Cristóbal & 827938.3 & 1186292.7 & 1870.5 & Lluvia & sep-11 & -9.37 & -64.8 & 10.2 \\
\hline CRI_PL & San Cristóbal & 827938.3 & 1186292.7 & 1870.5 & Lluvia & oct-11 & -13.07 & -94.2 & 10.4 \\
\hline CRI_PL & San Cristóbal & 827938.3 & 1186292.7 & 1870.5 & Lluvia & nov-11 & -14.45 & -105.7 & 9.9 \\
\hline CRI_PL & San Cristóbal & 827938.3 & 1186292.7 & 1870.5 & Lluvia & dic-11 & -11.24 & -78.9 & 11.0 \\
\hline CRI_PL & San Cristóbal & 827938.3 & 1186292.7 & 1870.5 & Lluvia & ene-12 & -6.16 & -39.3 & 10.0 \\
\hline CRI_PL & San Cristóbal & 827938.3 & 1186292.7 & 1870.5 & Lluvia & feb-12 & -7.75 & -51.8 & 10.2 \\
\hline CRI_PL & San Cristóbal & 827938.3 & 1186292.7 & 1870.5 & Lluvia & mar-12 & -7.73 & -53.6 & 8.3 \\
\hline CRI_PL & San Cristóbal & 827938.3 & 1186292.7 & 1870.5 & Lluvia & $a b r-12$ & -13.49 & -97.9 & 10.0 \\
\hline CRI_PL & San Cristóbal & 827938.3 & 1186292.7 & 1870.5 & Lluvia & may-12 & -15.69 & -118.2 & 7.3 \\
\hline CRI_PL & San Cristóbal & 827938.3 & 1186292.7 & 1870.5 & Lluvia & jun-12 & -11.17 & -82.3 & 7.0 \\
\hline CRI_PL & San Cristóbal & 827938.3 & 1186292.7 & 1870.5 & Lluvia & jul-12 & -10.93 & -80.1 & 7.3 \\
\hline CRI_PL & San Cristóbal & 827938.3 & 1186292.7 & 1870.5 & Lluvia & ago-12 & -7.56 & -51.1 & 9.4 \\
\hline CRI_PL & San Cristóbal & 827938.3 & 1186292.7 & 1870.5 & Lluvia & sep-12 & -5.50 & -35.4 & 8.5 \\
\hline CRI_PL & San Cristóbal & 827938.3 & 1186292.7 & 1870.5 & Lluvia & oct-12 & -9.79 & -67.5 & 10.9 \\
\hline CRI_PL & San Cristóbal & 827938.3 & 1186292.7 & 1870.5 & Lluvia & nov-12 & -7.83 & -48.5 & 14.1 \\
\hline CRI_PL & San Cristóbal & 827938.3 & 1186292.7 & 1870.5 & Lluvia & dic-12 & -5.26 & -30.2 & 11.9 \\
\hline CRI_PL & San Cristóbal & 827938.3 & 1186292.7 & 1870.5 & Lluvia & ene-13 & -0.50 & 6.3 & 10.3 \\
\hline CRI_PL & San Cristóbal & 827938.3 & 1186292.7 & 1870.5 & Lluvia & feb-13 & -2.67 & -7.8 & 13.5 \\
\hline CRI_PL & San Cristóbal & 827938.3 & 1186292.7 & 1870.5 & Lluvia & mar-13 & -6.17 & -39.1 & 10.3 \\
\hline FEL_PL & San Félix & 831886.5 & 1192132.7 & 2381 & Lluvia & mar-11 & -7.88 & -51.6 & 11.4 \\
\hline FEL_PL & San Félix & 831886.5 & 1192132.7 & 2381 & Lluvia & $a b r-11$ & -14.56 & -104.5 & 12.0 \\
\hline FEL_PL & San Félix & 831886.5 & 1192132.7 & 2381 & Lluvia & jul-11 & -12.36 & -91.2 & 7.7 \\
\hline FEL_PL & San Félix & 831886.5 & 1192132.7 & 2381 & Lluvia & ago-11 & -12.50 & -88.4 & 11.6 \\
\hline FEL PL & San Félix & 831886.5 & 1192132.7 & 2381 & Lluvia & sep-11 & -10.56 & -72.2 & 12.3 \\
\hline FEL_PL & San Félix & 831886.5 & 1192132.7 & 2381 & Lluvia & oct-11 & -13.86 & -98.3 & 12.6 \\
\hline FEL_PL & San Félix & 831886.5 & 1192132.7 & 2381 & Lluvia & nov-11 & -15.23 & -110.2 & 11.6 \\
\hline FEL_PL & San Félix & 831886.5 & 1192132.7 & 2381 & Lluvia & dic-11 & -11.16 & -76.9 & 12.4 \\
\hline FEL_PL & San Félix & 831886.5 & 1192132.7 & 2381 & Lluvia & feb-12 & -5.82 & -32.9 & 13.6 \\
\hline FEL_PL & San Félix & 831886.5 & 1192132.7 & 2381 & Lluvia & mar-12 & -9.60 & -65.4 & 11.4 \\
\hline FEL_PL & San Félix & 831886.5 & 1192132.7 & 2381 & Lluvia & $a b r-12$ & -12.99 & -93.0 & 10.9 \\
\hline FEL_PL & San Félix & 831886.5 & 1192132.7 & 2381 & Lluvia & may-12 & -15.68 & -116.0 & 9.5 \\
\hline FEL PL & San Félix & 831886.5 & 1192132.7 & 2381 & Lluvia & jul-12 & -11.37 & -81.9 & 9.1 \\
\hline FEL PL & San Félix & 831886.5 & 1192132.7 & 2381 & Lluvia & ago-12 & -8.83 & -58.1 & 12.6 \\
\hline FEL_PL & San Félix & 831886.5 & 1192132.7 & 2381 & Lluvia & sep-12 & -6.62 & -40.5 & 12.5 \\
\hline FEL_PL & San Félix & 831886.5 & 1192132.7 & 2381 & Lluvia & oct-12 & -12.35 & -88.4 & 10.4 \\
\hline FEL_PL & San Félix & 831886.5 & 1192132.7 & 2381 & Lluvia & nov-12 & -8.23 & -51.7 & 14.2 \\
\hline FEL_PL & San Félix & 831886.5 & 1192132.7 & 2381 & Lluvia & dic-12 & -5.94 & -33.8 & 13.7 \\
\hline FEL_PL & San Félix & 831886.5 & 1192132.7 & 2381 & Lluvia & ene-13 & -1.84 & 0.7 & 15.4 \\
\hline FEL_PL & San Félix & 831886.5 & 1192132.7 & 2381 & Lluvia & feb-13 & -3.50 & -13.8 & 14.2 \\
\hline FEL_PL & San Félix & 831886.5 & 1192132.7 & 2381 & Lluvia & mar-13 & -5.04 & -27.1 & 13.3 \\
\hline HEL_PL & Santa Elena & 843182 & 1177312 & 2607 & Lluvia & mar-11 & -9.37 & -63.4 & 11.6 \\
\hline HEL_PL & Santa Elena & 843182 & 1177312 & 2607 & Lluvia & $a b r-11$ & -16.55 & -120.2 & 12.2 \\
\hline HEL PL & Santa Elena & 843182 & 1177312 & 2607 & Lluvia & may-11 & -18.88 & -139.8 & 11.2 \\
\hline HEL_PL & Santa Elena & 843182 & 1177312 & 2607 & Lluvia & jun-11 & -14.97 & -109.7 & 10.0 \\
\hline HEL_PL & Santa Elena & 843182 & 1177312 & 2607 & Lluvia & jul-11 & -12.13 & -87.4 & 9.6 \\
\hline HEL_PL & Santa Elena & 843182 & 1177312 & 2607 & Lluvia & ago-11 & -12.50 & -89.9 & 10.1 \\
\hline HEL_PL & Santa Elena & 843182 & 1177312 & 2607 & Lluvia & sep-11 & -9.81 & -65.5 & 13.0 \\
\hline HEL_PL & Santa Elena & 843182 & 1177312 & 2607 & Lluvia & oct-11 & -14.09 & -97.9 & 14.8 \\
\hline HEL_PL & Santa Elena & 843182 & 1177312 & 2607 & Lluvia & nov-11 & -15.31 & -110.6 & 11.9 \\
\hline HEL_PL & Santa Elena & 843182 & 1177312 & 2607 & Lluvia & dic-11 & -12.14 & -83.5 & 13.6 \\
\hline HEL_PL & Santa Elena & 843182 & 1177312 & 2607 & Lluvia & ene-12 & -5.36 & -29.1 & 13.8 \\
\hline HEL_PL & Santa Elena & 843182 & 1177312 & 2607 & Lluvia & feb-12 & -5.43 & -30.0 & 13.5 \\
\hline HEL_PL & Santa Elena & 843182 & 1177312 & 2607 & Lluvia & mar-12 & -10.55 & -72.1 & 12.4 \\
\hline HEL_PL & Santa Elena & 843182 & 1177312 & 2607 & Lluvia & $a b r-12$ & -15.56 & -112.8 & 11.7 \\
\hline HEL_PL & Santa Elena & 843182 & 1177312 & 2607 & Lluvia & may-12 & -16.48 & -122.7 & 9.1 \\
\hline HEL_PL & Santa Elena & 843182 & 1177312 & 2607 & Lluvia & jun-12 & -12.70 & -91.0 & 10.6 \\
\hline HEL_PL & Santa Elena & 843182 & 1177312 & 2607 & Lluvia & jul-12 & -13.00 & -94.0 & 10.0 \\
\hline HEL_PL & Santa Elena & 843182 & 1177312 & 2607 & Lluvia & ago-12 & -9.30 & -62.0 & 12.4 \\
\hline HEL_PL & Santa Elena & 843182 & 1177312 & 2607 & Lluvia & sep-12 & -7.00 & -43.0 & 13.0 \\
\hline HEL_PL & Santa Elena & 843182 & 1177312 & 2607 & Lluvia & oct-12 & -13.40 & -94.0 & 13.2 \\
\hline HEL_PL & Santa Elena & 843182 & 1177312 & 2607 & Lluvia & nov-12 & -10.10 & -65.0 & 15.8 \\
\hline HEL_PL & Santa Elena & 843182 & 1177312 & 2607 & Lluvia & dic-12 & -6.90 & -38.0 & 17.2 \\
\hline HEL_PL & Santa Elena & 843182 & 1177312 & 2607 & Lluvia & ene-13 & -2.50 & -1.0 & 19.0 \\
\hline HEL_PL & Santa Elena & 843182 & 1177312 & 2607 & Lluvia & feb-13 & -3.77 & -16.9 & 13.3 \\
\hline HEL_PL & Santa Elena & 843182 & 1177312 & 2607 & Lluvia & mar-13 & -5.87 & -34.2 & 12.7 \\
\hline DOM_PL & Santo Domingo & 837726.6 & 1188102.3 & 1891 & Lluvia & mar-11 & -8.42 & -60.4 & 7.0 \\
\hline
\end{tabular}




\begin{tabular}{|c|c|c|c|c|c|c|c|c|c|}
\hline Código & Nombre & $\mathbf{X}$ & $\mathbf{Y}$ & Cota & tipo de muestra & Fecha & $18^{\circ}$ & $2 \mathrm{H}$ & d \\
\hline DOM_PL & Santo Domingo & 837726.6 & 1188102.3 & 1891 & Lluvia & abr-11 & -12.75 & -93.5 & 8.5 \\
\hline DOM_PL & Santo Domingo & 837726.6 & 1188102.3 & 1891 & Lluvia & may-11 & -17.85 & -132.0 & 10.8 \\
\hline DOM_PL & Santo Domingo & 837726.6 & 1188102.3 & 1891 & Lluvia & jun-11 & -14.40 & -106.3 & 8.9 \\
\hline DOM_PL & Santo Domingo & 837726.6 & 1188102.3 & 1891 & Lluvia & jul-11 & -11.52 & -83.8 & 8.4 \\
\hline DOM_PL & Santo Domingo & 837726.6 & 1188102.3 & 1891 & Lluvia & ago-11 & -12.20 & -88.0 & 9.6 \\
\hline DOM_PL & Santo Domingo & 837726.6 & 1188102.3 & 1891 & Lluvia & sep-11 & -8.82 & -60.5 & 10.1 \\
\hline DOM_PL & Santo Domingo & 837726.6 & 1188102.3 & 1891 & Lluvia & oct-11 & -13.47 & -98.1 & 9.7 \\
\hline DOM_PL & Santo Domingo & 837726.6 & 1188102.3 & 1891 & Lluvia & nov-11 & -15.05 & -111.1 & 9.3 \\
\hline DOM_PL & Santo Domingo & 837726.6 & 1188102.3 & 1891 & Lluvia & dic-11 & -11.37 & -78.5 & 12.5 \\
\hline DOM_PL & Santo Domingo & 837726.6 & 1188102.3 & 1891 & Lluvia & ene-12 & -8.51 & -59.0 & 9.1 \\
\hline DOM_PL & Santo Domingo & 837726.6 & 1188102.3 & 1891 & Lluvia & feb-12 & -5.13 & -33.9 & 7.1 \\
\hline DOM_PL & Santo Domingo & 837726.6 & 1188102.3 & 1891 & Lluvia & mar-12 & -8.35 & -59.7 & 7.1 \\
\hline DOM_PL & Santo Domingo & 837726.6 & 1188102.3 & 1891 & Lluvia & $a b r-12$ & -12.63 & -91.6 & 9.5 \\
\hline DOM_PL & Santo Domingo & 837726.6 & 1188102.3 & 1891 & Lluvia & may-12 & -15.91 & -120.1 & 7.2 \\
\hline DOM_PL & Santo Domingo & 837726.6 & 1188102.3 & 1891 & Lluvia & ago-12 & -7.83 & -54.6 & 8.1 \\
\hline DOM_PL & Santo Domingo & 837726.6 & 1188102.3 & 1891 & Lluvia & oct-12 & -12.95 & -93.65 & 9.9 \\
\hline DOM_PL & Santo Domingo & 837726.6 & 1188102.3 & 1891 & Lluvia & nov-12 & -8.10 & -54.8 & 10.0 \\
\hline DOM_PL & Santo Domingo & 837726.6 & 1188102.3 & 1891 & Lluvia & dic-12 & -6.54 & -39.4 & 13.0 \\
\hline DOM_PL & Santo Domingo & 837726.6 & 1188102.3 & 1891 & Lluvia & ene-13 & -1.14 & 0.8 & 9.9 \\
\hline DOM_PL & Santo Domingo & 837726.6 & 1188102.3 & 1891 & Lluvia & feb-13 & -2.19 & -7.6 & 9.9 \\
\hline DOM_PL & Santo Domingo & 837726.6 & 1188102.3 & 1891 & Lluvia & mar-13 & -4.98 & -27.9 & 12.0 \\
\hline UDE_PL & UdeA & 835033 & 1185159 & 1491 & Lluvia & mar-11 & -6.54 & -42.8 & 9.5 \\
\hline UDE_PL & UdeA & 835033 & 1185159 & 1491 & Lluvia & may-11 & -16.53 & -123.2 & 9.0 \\
\hline UDE_PL & UdeA & 835033 & 1185159 & 1491 & Lluvia & jun-11 & -13.63 & -101.8 & 7.2 \\
\hline UDE_PL & UdeA & 835033 & 1185159 & 1491 & Lluvia & ago-11 & -11.03 & -80.8 & 7.4 \\
\hline UDE_PL & UdeA & 835033 & 1185159 & 1491 & Lluvia & sep-11 & -9.01 & -63.1 & 9.0 \\
\hline UDE_PL & UdeA & 835033 & 1185159 & 1491 & Lluvia & oct-11 & -12.86 & -93.6 & 9.3 \\
\hline UDE_PL & UdeA & 835033 & 1185159 & 1491 & Lluvia & nov-11 & -14.71 & -109.6 & 8.1 \\
\hline UDE_PL & UdeA & 835033 & 1185159 & 1491 & Lluvia & dic-11 & -10.51 & -74.6 & 9.5 \\
\hline UDE_PL & UdeA & 835033 & 1185159 & 1491 & Lluvia & ene-12 & -3.20 & -11.0 & 14.6 \\
\hline UDE_PL & UdeA & 835033 & 1185159 & 1491 & Lluvia & feb-12 & -3.76 & -26.8 & 3.3 \\
\hline UDE_PL & UdeA & 835033 & 1185159 & 1491 & Lluvia & mar-12 & -6.42 & -44.5 & 6.9 \\
\hline UDE_PL & UdeA & 835033 & 1185159 & 1491 & Lluvia & abr-12 & -12.05 & -87.4 & 9.0 \\
\hline UDE_PL & UdeA & 835033 & 1185159 & 1491 & Lluvia & may-12 & -15.26 & -115.3 & 6.8 \\
\hline UDE_PL & UdeA & 835033 & 1185159 & 1491 & Lluvia & jul-12 & -10.50 & -77.0 & 7.0 \\
\hline UDE_PL & UdeA & 835033 & 1185159 & 1491 & Lluvia & ago-12 & -6.90 & -44.0 & 11.2 \\
\hline UDE_PL & UdeA & 835033 & 1185159 & 1491 & Lluvia & sep-12 & -5.30 & -30.0 & 12.4 \\
\hline UDE_PL & UdeA & 835033 & 1185159 & 1491 & Lluvia & oct-12 & -11.40 & -81.0 & 10.2 \\
\hline UDE_PL & UdeA & 835033 & 1185159 & 1491 & Lluvia & nov-12 & -8.30 & -52.0 & 14.4 \\
\hline UDE_PL & UdeA & 835033 & 1185159 & 1491 & Lluvia & dic-12 & -14.60 & -107.0 & 9.8 \\
\hline UDE_PL & UdeA & 835033 & 1185159 & 1491 & Lluvia & ene-13 & -3.20 & -11.0 & 14.6 \\
\hline UDE_PL & UdeA & 835033 & 1185159 & 1491 & Lluvia & feb-13 & -1.64 & -4.2 & 14.6 \\
\hline UDE_PL & UdeA & 835033 & 1185159 & 1491 & Lluvia & mar-13 & -5.12 & -31.3 & 14.6 \\
\hline UDE_PL & UdeA & 835033 & 1185159 & 1491 & Lluvia & mar-17 & -7.43 & -52.7 & 6.71 \\
\hline UDE_PL & UdeA & 835033 & 1185159 & 1491 & Lluvia & abr-17 & -10.41 & -77.2 & 6.12 \\
\hline UDE_PL & UdeA & 835033 & 1185159 & 1491 & Lluvia & jun-17 & -12.67 & -97.3 & 4.1 \\
\hline UDE_PL & UdeA & 835033 & 1185159 & 1491 & Lluvia & jul-17 & -10.65 & -79.2 & 6.0 \\
\hline UDE_PL & UdeA & 835033 & 1185159 & 1491 & Lluvia & ago-17 & -7.55 & -52.6 & 7.8 \\
\hline SPR_02 & Fuente ecológico & 6.263267 & -75.49969 & 2445.27 & Lluvia & mar-17 & -8.57 & -59.3 & 9.2 \\
\hline SPR_02 & Fuente ecológico & 6.263267 & -75.49969 & 2445.27 & Lluvia & $a b r-17$ & -10.02 & -72.7 & 7.4 \\
\hline SPR_02 & Fuente ecológico & 6.263267 & -75.49969 & 2445.27 & Lluvia & may-17 & -18.82 & -142.0 & 8.6 \\
\hline SPR_02 & Fuente ecológico & 6.263267 & -75.49969 & 2445.27 & Lluvia & jun-17 & -15.04 & -113.4 & 6.9 \\
\hline SPR_02 & Fuente ecológico & 6.263267 & -75.49969 & 2445.27 & Lluvia & jul-17 & -12.84 & -92.2 & 10.5 \\
\hline SPR_02 & Fuente ecológico & 6.263267 & -75.49969 & 2445.27 & Lluvia & ago-17 & -8.69 & -58.9 & 10.6 \\
\hline SPR_03 & Colegio new & 6.18881 & -75.545749 & 2144.11 & Lluvia & mar-17 & -8.34 & -58.2 & 8.5 \\
\hline SPR_03 & Colegio new & 6.18881 & -75.545749 & 2144.11 & Lluvia & $a b r-17$ & -9.65 & -69.3 & 7.9 \\
\hline SPR_03 & Colegio new & 6.18881 & -75.545749 & 2144.11 & Lluvia & may-17 & -18.64 & -142.1 & 7.0 \\
\hline SPR_03 & Colegio new & 6.18881 & -75.545749 & 2144.11 & Lluvia & jun-17 & -13.97 & -105.1 & 6.6 \\
\hline SPR_03 & Colegio new & 6.18881 & -75.545749 & 2144.11 & Lluvia & jul-17 & -12.59 & -94.4 & 6.3 \\
\hline SPR_03 & Colegio new & 6.18881 & -75.545749 & 2144.11 & Lluvia & ago-17 & -8.24 & -56.0 & 9.9 \\
\hline SPR_04 & Colegio Débora Arango & 6.223499 & -75.625873 & 1671.58 & Lluvia & mar-17 & -6.13 & -41.1 & 7.9 \\
\hline SPR_04 & Colegio Débora Arango & 6.223499 & -75.625873 & 1671.58 & Lluvia & abr-17 & -9.02 & -65.2 & .7 .0 \\
\hline SPR_04 & Colegio Débora Arango & 6.223499 & -75.625873 & 1671.58 & Lluvia & may-17 & -18.22 & -139.9 & 6.0 \\
\hline SPR_04 & Colegio Débora Arango & 6.223499 & -75.625873 & 1671.58 & Lluvia & jul-17 & -10.99 & -82.6 & 5.4 \\
\hline SPR_04 & Colegio Débora Arango & 6.223499 & -75.625873 & 1671.58 & Lluvia & ago-17 & -7.02 & -49.4 & 6.7 \\
\hline SPR_05 & Seminario la Santa cruz & 6.090579 & -75.629997 & 1772.37 & Lluvia & mar-17 & -7.44 & -53.6 & 6.0 \\
\hline SPR_05 & Seminario la Santa cruz & 6.090579 & -75.629997 & 1772.37 & Lluvia & $a b r-17$ & -10.51 & -78.2 & 5.9 \\
\hline SPR_05 & Seminario la Santa cruz & 6.090579 & -75.629997 & 1772.37 & Lluvia & may-17 & -17.69 & -134.9 & 6.6 \\
\hline SPR_05 & Seminario la Santa cruz & 6.090579 & -75.629997 & 1772.37 & Lluvia & jul-17 & -12.32 & -91.5 & 7.1 \\
\hline SPR_05 & Seminario la Santa cruz & 6.090579 & -75.629997 & 1772.37 & Lluvia & ago-17 & -6.97 & -45.8 & 10.0 \\
\hline
\end{tabular}

Tabla 2. Información isotópica $(\delta 180$ y $\delta 2 \mathrm{H})$ en el agua lluvia.

Table 2. $(\delta 180$ y $\delta 2 H)$ in precipitation. 


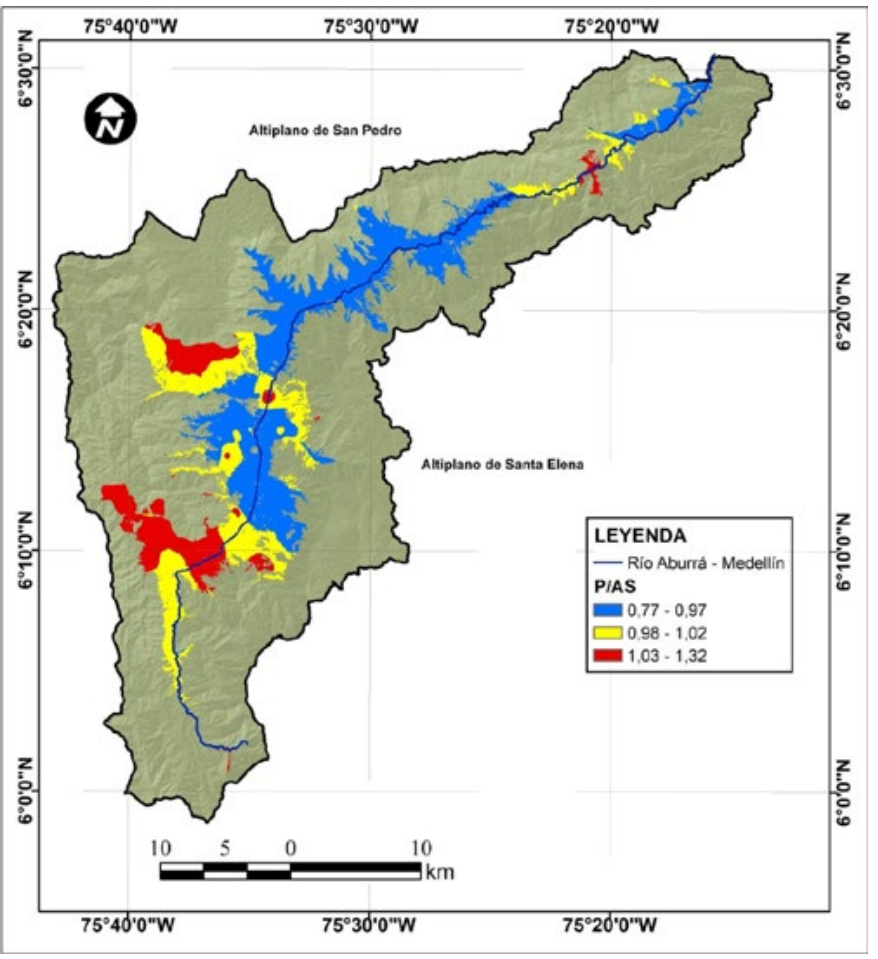

Figura 8. Relación P/AS para el $\delta^{18} \mathrm{O}$ en el Valle de Aburrá.

Figure 8. P/AS ratio of $\delta^{18} \mathrm{O}$ of the Aburrá Valley.

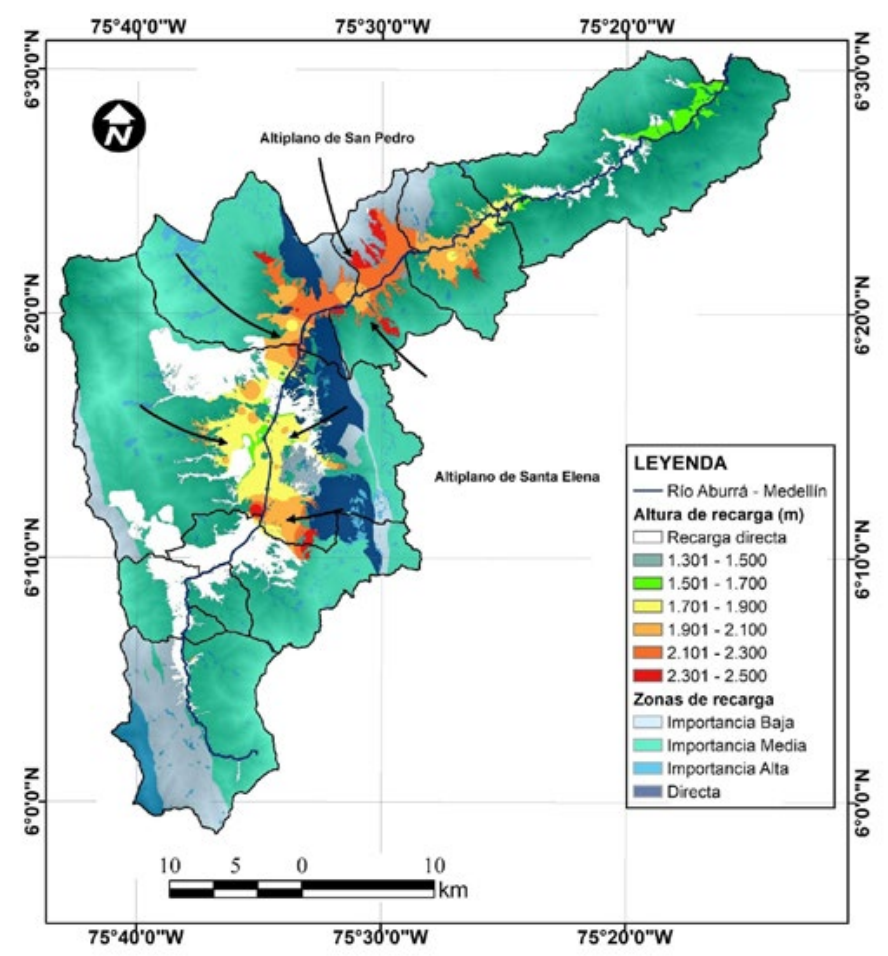

Figura 9. Relación entre zonas de recarga directa e indirecta de importancia alta con la relación isotópica de la recarga.

Figure 9. Relationship between areas of direct and indirect recharge of high importance with the isotopic ratio of recharge. que estarían teniendo lugar por fuera de la frontera de la zona de recarga directa, insinuando la probable ocurrencia de flujos intermedios o regionales, responsables de la presencia de agua subterránea en el acuífero en la zona más intervenida por procesos de impermeabilización a causa de la expansión de la urbanización. Si se relacionan, para esta zona los registros de $\delta^{18} \mathrm{O}$ y $\delta^{2} \mathrm{H}$ del agua subterránea con la variación altitudinal de la precipitación, se detecta la correspondencia con la de lluvias que precipitan por encima de 1500 m s.n.m y hasta 2600 metros de altura.

Respecto a la datación del agua subterránea en el acuífero Libre del Valle de Aburrá, ya a partir de análisis anteriores (Área Metropolitana del Valle de Aburrá y Universidad de Antioquia, 2016), en los que se había analizado la evolución hidrogeoquímica de las aguas subterráneas, se tenían indicios de tiempos de residencia largos, esto relacionado con la presencia de aguas en las que se registran valores de conductividad eléctrica por encima de $2000 \mu \mathrm{S} / \mathrm{cm}$ en muestras bicarbonatadas magnésicas y cloruradas sódicas (Área Metropolitana del Valle de Aburrá y Universidad de Antioquia. 2013; Campillo et al., 2015). Tres de los valores reportados para ${ }^{3} \mathrm{H}$ en el agua subterránea del acuífero libre fueron 1,1 $( \pm 0,3)$ UT, $1,0( \pm 0,3)$ UT y mínimo $0,9( \pm 0,3)$ UT, indicadores de aguas recargadas recientemente si se tiene en cuenta que en la Iluvia se midieron 0,9 UT. Una sola muestra tendría valores menores al límite de detección del laboratorio $(0,8$ UT), lo que indicaría aguas de más de 60 años. La información con que se cuenta para ${ }^{14} \mathrm{C}$ no permite precisar mejor la edad, debido a que la datación corresponde a muestras provenientes de mezclas de agua del acuífero libre y el acuífero semiconfinado.

\section{Discusión}

Considerando el modelo hidrogeológico conceptual que se tiene para el sistema Acuífero del Valle de Aburrá y teniendo en cuenta para él los componentes mejor explorados y conocidos, esto es: el acuífero libre y la delimitación de potenciales zonas de recarga asociadas a sistemas de flujo, la utilización de datos isotópicos y su modelación mediante mapas isotópicos permiten establecer una mayor certeza acerca de los procesos de flujo subterráneo.

En la Figura 9 se presenta el mapa producto de la superposición de las zonas de recarga del acuífero del Valle de Aburrá (Figura 2), con el resultado que se obtiene de reconstruir a partir del mapa de $\delta^{18} \mathrm{O}$ en las aguas subterráneas y gradiente altitudinal la altura $\left(\delta^{18} \mathrm{O}=-0,0014 \mathrm{~h}-8,837\right)$ a la que se estaría produciendo la recarga para el valle en aquellas superficies donde la relación que se obtuvo para P/AS fue menor que 1.

Teniendo en cuenta este resultado, se observan tres situaciones de interés:

i) Recarga directa: existen algunas superficies, den- 
tro del Valle de Aburrá, donde la recarga al acuífero libre se estaría dando directamente a partir de la lluvia eficaz que es la que produce recarga. Esta situación se reconoce en el sur, en el sector centro occidental y puntualmente al norte.

ii) Fuentes de recarga asociadas a flujos intermedios: situación que se presenta espacialmente en el centro del valle. En la margen occidental la recarga provendría de áreas de recarga indirecta de importancia alta, ubicadas entre 1700 m s.n.m y 2300 m s.n.m. Desde el oriente se produciría flujo al acuífero desde la Dunita de Medellín. Ya los análisis hidrogeoquímicos realizados en estudios anteriores habían revelado la naturaleza magnésica de las aguas subterráneas en esta zona del Valle (Área Metropolitana del Valle de Aburrá y Universidad de Antioquia, 2013).

iii) Flujos Regionales: se identifica esta situación después de que el río Medellín cambia su curso sur-norte hacia el noreste, justo donde el valle se torna de nuevo estrecho, la información isotópica que se deriva de las aguas subterráneas en ese tramo -donde las rocas encajantes corresponden, por sus condiciones geomorfológicas y estructurales a condiciones de recarga indirecta de importancia media a baja- indica la ocurrencia de fuentes localizadas por fuera de la zona de estudio, a alturas que alcanzarían 2.500 m s.n.m. Esto indicaría flujos regionales desde otras cuencas o desde los altiplanos de San Pedro al Norte y Santa Elena al oriente.

Estas tres situaciones ponen de manifiesto que en el área urbanizada del Valle de Aburrá, el agua almacenada en el acuífero no proviene de recarga directa sino de flujos intermedios y regionales, situación que pone en evidencia el impacto de la impermeabilización urbana sobre la sostenibilidad del agua subterránea.

Es importante señalar que los resultados que se obtienen, respecto a la distribución espacial, tanto en los mapas isotópicos de precipitación y agua subterránea como en el de la relación P/AS, permiten identificar variaciones tendenciales bien definidas o extensiones que sugieren sectores con condiciones homogéneas. Esta distribución, con patrones claramente definidos, se considera un indicador de la validez de los análisis, especialmente si se tiene en consideración que la zona de estudio registra patrones de circulación atmosférica complejos en los que se combinan procesos propios de la Zona Intertropical de Convergencia, efectos de inversión térmica y patrones de circulación valle montaña (Rendón, 2014). Tal y como se ha evidenciado en otros estudios realizados en regiones montañosas tropicales (Gómez et al., 2015 y Gonfiantini et al., 2001), estas circunstancias podrían ser las determinantes de la magnitud que registra el gradiente altitudinal.

Finalmente, teniendo en cuenta las relaciones para la LMRA $\left(\delta^{2} \mathrm{H}=8,09 \delta^{18} \mathrm{O}+10,9 \%\right.$. $)$ y la LML $\left(\delta^{2} \mathrm{H}=\right.$ $8,23 \delta^{18} \mathrm{O}+12,2 \%$ ) y los respectivos gradientes altitudinales $(0,17 \%$ o cada 100 metros 0,14 y \%o cada 100 metros), se plantea como hipótesis que la similitud y ajuste entre ambos modelos (Regional y Local) permite establecer que mapa isotópico regional de precipitación para Antioquia puede constituir una representación aceptable de la variabilidad espacial, de la naturaleza isotópica de la lluvia y por lo tanto, este mapa, representa es un primer insumo a utilizarse para aproximar la localización de zonas de recarga a otros sistemas hidrogeológicos locales en los que se disponga de datos para $\delta^{18} \mathrm{O}$ y $\delta^{2} \mathrm{H}$ en el agua subterránea.

\section{Conclusiones}

Este primer ejercicio de construcción de mapas isotópicos en Colombia, que relaciona mapas isotópicos de lluvia y agua subterránea, constituye un indicador general del comportamiento de la recarga directa y la existencia de flujos regionales que soportan la sostenibilidad de los niveles y reservorios de agua subterránea.

El desarrollo de este trabajo permitió evaluar para el Valle de Aburrá, en el departamento de Antioquia Colombia, la distribución de la composición isotópica de la precipitación en cuanto a los isótopos estables ${ }^{18} \mathrm{O}$ y ${ }^{2} \mathrm{H}$ y obtener su distribución espacial. El análisis de esta distribución de la precipitación, sumado al de la distribución espacial de los isótopos en el agua subterránea, es una herramienta para evaluar los mecanismos de recarga del agua subterránea, que posteriormente deberán ser objeto de medidas de manejo para su protección, especialmente en un valle urbanizado donde es evidente el aporte por flujos regionales al acuífero.

Los resultados de este trabajo han sido comparados con el modelo hidrogeológico del Valle de Aburrá, en el que se tiene la delimitación de los acuíferos y las zonas de recarga. Lográndose identificar recarga directa, flujos intermedios y regionales.

Se considera necesario que a futuro se establezcan relaciones con variables climatológicas, como humedad relativa, temperatura y dirección de los vientos, con el fin de obtener mapas isotópicos con variables que puedan ser susceptibles a cambios en el tiempo y el espacio. Además, la representación espacial de la composición isotópica de la lluvia y del agua subterránea ayuda a definir criterios para establecer la necesidad de realizar datación isotópica, principalmente donde se observan sistemas de flujos regionales.

\section{Agradecimientos}

Este trabajo se desarrolla gracias a los recursos del Contrato 22326 entre la Universidad de Antioquia y el Organismo Internacional de Energía Atómica. Además, los autores agradecen al Área Metropolitana del Valle de Aburrá - AMVA -, entidad con la que se realiza desde 2010 el monitoreo hidrogeológico de la región. 


\section{Referencias}

Área Metropolitana del Valle de Aburrá y Universidad de Antioquia. 2013. Determinación de las Potenciales Zonas de Recarga en el Centro y Sur del Valle de Aburrá.

Área Metropolitana del Valle de Aburrá y Universidad de Antioquia. 2016. Red de Monitoreo AMbiental en la Cuenca Hidrográfica del Río Aburrá en Jurisdicción del Área Metropolitana. Fase V. Convenio 368 de 2014. Operación de Red de Aguas Subterráneas. https://www.metropol.gov.co/ambiental/recurso-hidrico/aguassubterraneas/fase-v-aguas-subterraneas.pdf

Betancur, T. Martínez, C. García, E. F., Escobar J.F. 2017. Identification and characterization of regional water flows contributing to the recharge of an unconfined aquifer. Revista Facultad de Ingeniería, 85, 70-85.

Campillo, A. 2012. Orígenes de la recarga de los acuíferos del Valle de Aburrá. Tesis de Maestría, Universidad de Montpellier, 58pp.

Campillo, A., Taupin, J., Patris, N., Betancur T., 2015. Uso de la geoquímica y de los isótopos estables del agua en el estudio de un sistema acuífero superficial en el complejo urbanizado andino (Valle de Aburrá-Colombia). Revista peruana geo-atmosférica RPGA, 4, 62-79

Cano, A. 2015. Identificación de la presencia de flujos regionales en el sistema hidrogeológico del Valle de Aburrá a partir de un caso de estudio en una zona piloto. Tesis de Maestría, Universidad de Antioquia.

Clark, I. D., Fritz, P. 1997. Environmental Isotopes in Hydrogeology. Lewis Publishers, 328 pp, New York

Craig, H. 1961. Isotopic variations in meteoric waters, Science, 133(3465), 1702-1703.

Dansgaard, W. 1964. Stable isotopes in precipitation, Tellus, 16, 436-468.

Escobar, J. Betancur, T. García E. Martínez, C., Palacio, P. 2017. Análisis jerárquico ponderado aplicado a la identificación de recarga y flujos regionales en acuíferos. Revista Politécnica, 24, 37-48.

Gómez, S., Taupin, J . Rueda, J., 2015. Estudio hidrodinámico, geoquímico e isotópico de las formacio- nes acuíferas de la región de Bucaramanga (Colombia). RGPA. pp. 44-61.

Gonfiantini R., Roche M-A., Olivry J., Fontes J. y Zuppi G. 2001, The altitude effect on the isotopic composition of tropical rains. Chemical Geology 1812001 147-167, 2001

IDEAM. 2018. Estudio Nacional del Agua. Bogotá, 2018.

Langston, G., Bentley, L. R., Hayashi, M., McClymont, A., \& Pidlisecky, A. (2011). Internal structure and hydrological functions of an alpine proglacial moraine. Hydrological processes, 25(19), 2967-2982.

Londoño L., Ramírez J., Ramírez G. y Ordoñez C., 2010. Modelo de Interpolación Espacial Mediante Sistemas de Información Geográfica para el Estudio de la Variabilidad Espacial de la Resistencia a la Penetración del Suelo. Revista DYNA, vol. 77, No. 164

Mook, W. G., 2002. Isótopos ambientales en el ciclo del agua. Principios y Aplicaciones. Publicación del IGME

Ossa J., Campillo A., Omar C., Betancur T. 2018. Análisis regional de la recarga del agua subterránea a partir de isoscapes de precipitación. Caso de estudio: Antioquia, Colombia. In: Rodolfo Fernando García [et al.] (ed.), El Agua Subterránea: Recurso sin Fronteras: Hidrogeología Regional. Volumen I. Argentina, 175-182.

Rendon A., 2014. Effects of Urbanization on theTemperature Inversion Breakup in a Mountain Valley with Implications for Air Quality. Journal of applied meteorology and climatology, 53(4), $840-858$.

Sánchez-Murillo, R., Birkel, C. 2016. Groundwater recharge mechanisms inferred from isoscapes in a complex tropical mountainous region. Geophysical Research Letters, 43(10), 5060-5069.

Wassenaar, L. I., Van Wilgenburg, S. L., Larson, K., Hobson, K. A. 2009. A groundwater isoscape ( $\delta D$, ס180) for Mexico, Journal of Geochemical Exploration., 102(3), 123-136.

Yamanaka, T., Makino, Y., Wakiyama, Y., Kishi, K., Maruyama, K., Kano, M., Ma, W., Suzuki, K. 2015. How reliable are modeled precipitation isoscapes over a high-relief mountainous region?, Hydrol. Res. Lett., 9(4), 118-124.

Recibido: julio 2019

Revisado: noviembre 2019

Aceptado: enero 2020

Publicado: marzo 2021 
